\title{
Comments on: Epidemiological study of urge urinary incontinence and risk factors in China
}

\author{
Hai Li $\cdot$ Xiangbo Kong $\cdot$ Hong-Liang Zhang $\cdot$ Jiang Wu
}

Received: 14 March 2010 / Accepted: 11 April 2010 / Published online: 19 August 2010

(C) The International Urogynecological Association 2010

\section{Dear Editor,}

Zhu and colleagues investigated urge urinary incontinence (UUI) and risk factors in China via a cross-sectional study involving 20,000 adult Chinese women [1]. This crossprovince survey is intriguing in that the authors provide us with a general profile of UUI among the general population in China. However, we are concerned about their interpretation of their findings.

As many potential risks factors were not evaluated in the study, the conclusion that age is a major independent risk factor of UUI as draw in the article would raise concerns. The diagnosis of UUI is mainly based on clinical symptoms. Thus UUI can be viewed as a clinical syndrome. A variety of diseases may present symptoms like UUI, and many risk factors may contribute to the prevalence of UUI [2]. Limited by the methodological pitfall, this study failed to determine the causal relationships between UUI and risk factors. We agree that UUI should not be accepted as a natural consequence of aging and being untreatable. Therefore, patients with UUI, especially those who have atypical features or severe symptoms, should appeal to

\section{H. Li $\cdot$ X. Kong}

Department of Urology,

China-Japan Union Hospital of Jilin University,

Changchun, China

\section{H.-L. Zhang $\cdot$ J. Wu}

Department of Neurology, The First Hospital of Jilin University,

Changchun, China

\section{H.-L. Zhang $(\triangle)$}

Department of Neurobiology, Care Sciences and Society,

Karolinska Institute,

Novum, plan 5,

14186 Stockholm, Sweden

e-mail: Hongliang.Zhang@ki.se specialist and undergo full assessment with cystoscopy and urodynamic study if necessary [2].

In their study, severity assessment such as information about incontinence episode frequency was not reported. Although limited by the relatively sample size, it would be interesting if there is a positive correlation between UUI severity and age, which might support age as a major risk factor indirectly.

Besides the common risk factors of UUI like gynecological diseases, urological disorders, as well as neurological disorders, such as cerebrovascular diseases might account for the causes of UUI. In the light of this consideration, urological symptoms such as urodynia, hematuria, or neurological symptoms which may point to a neuropathic bladder as the cause of UUI (e.g., new onset weakness, paraesthesia, back pain, visual disturbances) are of great value to clarify the etiology of UUI [3].

In addition, since it is a cross-province survey, we would like to know whether there is difference in the prevalence of UUI among provinces, as well as between rural and urban areas.

Acknowledgements The work was supported by grants from China Scholarship Council, Gun och Bertil Stohnes Foundation, and Stiftelsen för Gamla Tjänrinnor.

\section{References}

1. Zhu L, Lang J, Liu C, Xu T, Liu X, Li L et al (2010) Epidemiological study of urge urinary incontinence and risk factors in China. Int Urogynecol J DOI 10.1007/s00192-009-1041-4

2. Norton P, Brubaker L (2006) Urinary incontinence in women. Lancet 367:57-67

3. McKertich K (2008) Urinary incontinence - assessment in women: stress, urge or both? Aust Fam Physician 37:112-117 